\title{
Caracterización Físico-Química Y Organoléptica De Un Licor A Base De Piñuela
}

\author{
Arianna del Ángel Pacheco May, (Lic. En Gastronomía) \\ Bibiana Piedra Vázquez, (Lic. En Gastronomía) \\ Cecilia de Jesús López Velasco, (Lic. En Gastronomía) \\ Maximiliano Vanoye Eligio, (Doctor en Ciencias) \\ Jocabeth Eunice Naal Espinosa, (M.A.R.H)
}

Instituto Tecnológico Superior de Escárcega. Calle Col Unidad, Esfuerzo y

Trabajo No. 1. Escárcega, Campeche.

Doi:10.19044/esj.2022.v18n3p255

Submitted: 12 June 2021

Accepted: 20 August 2021

Published: 31 January 2022
Copyright 2022 Author(s)

Under Creative Commons BY-NC-ND 4.0 OPEN ACCESS

Cite As:

Pacheco May A., Vasques B.P., Velasco C., Eligio M.V.,\& Espinosa J.E.N.,(2022). Caracterización Físico-Química Y Organoléptica De Un Licor A Base De Piñuela European Scientific Journal, ESJ, 18 (3), 1.

https://doi.org/10.19044/esj.2022.v18n3p255

\section{Resumen}

La piñuela es una baya agridulce y jugosa, su pulpa es de color blanco. En la península de Yucatán, la población maya la conoce con distintos nombres, sin embargo, también ha sido registrado para al menos 13 estados del país. Esta especie crece en las regiones tropicales de ambos litorales en México, así como en Centroamérica y las islas del Caribe, presentando mayor abundancia en la zona costera y en la selva baja caducifolia. Los usos que se le da a la piñuela son diversos (ornamental, medicinal, fines alimenticios, decorativos y ceremonial). Por lo anterior, el objetivo de la presente investigación fue elaboración y caracterización de un licor del fruto de la piñuela (Bromelia pinguin L) de forma artesanal en el municipio de Escárcega, Campeche. Para el logro de la investigación se hizo la recolección del fruto en localidades rurales del municipio de Candelaria, las cuales fueron trasladas a las instalaciones del Instituto Tecnológico Superior de Escárcega para la elaboración del licor, en donde después de tres meses se procedió a la evaluación de aceptabilidad ante un panel semientrenado. Los resultados indican que presenta una alta concentración de alcohol (31). Los panelistas valoraron el licor de piñuela satisfactoriamente (más del 80\%). Como 
conclusión del estudio es importante considerar a este fruto como parte de un crecimiento en la industria alimentaria de la región, ya que por sus características naturales se le considera como un fuerte producto potencial para ser aprovechado por la comunidad local.

Mots clés: Piñuela, Licor, Físico-Química, Escárcega

\title{
Physical-Chemical And Organoleptic Characterization Of A Pineapple-Based Liquor
}

\author{
Arianna del Ángel Pacheco May, (Lic. En Gastronomía) \\ Bibiana Piedra Vázquez, (Lic. En Gastronomía) \\ Cecilia de Jesús López Velasco, (Lic. En Gastronomía) \\ Maximiliano Vanoye Eligio, (Doctor en Ciencias) \\ Jocabeth Eunice Naal Espinosa, (M.A.R.H) \\ Instituto Tecnológico Superior de Escárcega. Calle Col Unidad, Esfuerzo y \\ Trabajo No. 1. Escárcega, Campeche.
}

\section{Abstract}

The piñuela is a bittersweet and juicy berry, its pulp is white in color. In the Yucatan Peninsula, the Mayan population knows it by different names, however, it has also been recorded for at least 13 states in the country. This species grows in the tropical regions of both coasts in Mexico, as well as in Central America and the Caribbean islands, presenting greater abundance in the coastal zone and in the low deciduous forest. Piñuelas are used for a variety of purposes (ornamental, medicinal, food, decorative, and ceremonial). Therefore, the objective of this research was to elaborate and characterize a liqueur made from the fruit of the piñuela (Bromelia pinguin L) in an artisanal way in the municipality of Escárcega, Campeche. For the achievement of the research, the fruit was collected in rural localities of the municipality of Candelaria, which were transferred to the facilities of the Instituto Tecnológico Superior de Escárcega for the elaboration of the liquor, where after three months the evaluation of acceptability was made before a semitrained panel. The results indicate that it has a high alcohol concentration (31). The panelists rated the piñuela liqueur satisfactorily (more than $80 \%$ ). As a conclusion of the study, it is important to consider this fruit as part of a growing food industry in the region, since its natural characteristics make it a strong potential product to be exploited by the local community.

Keywords: Piñuela, Liquor, Physical-Chemical, Escárcega 


\section{Introducción}

La piñuela (Bromelia pinguin L.) es una baya agridulce y jugosa de cáscara hebrosa de color blanco amarillento a café oscuro, es completamente ferrugíneo, fusiforme, estrecho hacia la parte basal y apical, pero más redondo en su parte central. La pulpa es de color blanco dividida en tres lóculos con dos series de semillas de placentación parietal, ubicadas alternamente hacia el centro de color café a negro. En la península de Yucatán, la población maya la conoce como ch'am, ch' amch o, ch'om, jman, tslbay, ts'alvay. Sin embargo esto no quiere decir que se exclusiva del área, ya que ha sido registrado para al menos 13 estados del país (Chiapas, Colima, Guerrero, Jalisco, Michoacán, Nayarit, Oaxaca, Sinaloa, San Luis Potosí, Sonora, Tabasco, Tamaulipas y Veracruz), por lo que en distintas investigaciones se menciona que la especie crece en las regiones tropicales de ambos litorales en México, así como en Centroamérica y las islas del Caribe, presentando mayor abundancia en la zona costera y en la selva baja caducifolia (Rangel, 2009; Hornung-Leoni, 2011;).

Los usos que se le da a la piñuela son diversos (ornamental, medicinal, fines alimenticios, decorativos y ceremonial). Respecto al uso ceremonial, la población maya prepara una bebida ancestral llamada "Balché” a la cual se le agrega el fruto de piñuela que actúa como un conservador natural, sin la piñuela la bebida fermentada dura unas cuantas horas, hoy en día se puede degustar esta bebida y conservarla en casa. Entre otros usos comerciales que se le da a la piñuela en México destaca la obtención de su fibra utilizada en la elaboración de sacos, cordelería para adornar vestimenta de la charrería (cinturones, ropa con atuendos de cuero bordados con ella), costales de artesanía, redes, hamacas, bolsas de mano, huaraches (sandalias), cestas y hasta arcos de flechas (Sandoval Bucio et al., 2004). Igualmente en el sureste Nicaragua, se obtienen fibras y son tratadas con jugo de lima para reducir la oxidación y aclarar su color (Coe y Anderson, 1999). La fibra conocida bajo el nombre de "ixtle" o "fibra de pita" obtenida de Aechmea magdalenae y Bromelia pinguin es reconocida por su brillo, finura y resistencia, comparable a la de la seda, pero con mayor vigor (Edouard, 2005). Además de los usos que se han mencionado, también se ha señalado el uso de la piñuela en la elaboración de licor de esta fruta, mermelada y malvavisco. Por lo anterior, el objetivo de la presente investigación fue elaboración y caracterización de un licor del fruto de la piñuela (Bromelia pinguin L) de forma artesanal.

\section{Materiales y métodos}

Área de estudio

La recolección de los frutos para el estudio se llevó acabo en la región sur del estado de Campeche, específicamente en las comunidades Nuevo progreso II, Miguel Hidalgo y Costilla y el Sacrificio; por ser lugares donde 
de manera natural crece la piñuela. Esta región localizada en el sureste de la República Mexicana, cuenta con vegetación conocida como selva baja subcaducifolia y abundantes lluvias en verano. La región forma parte de una planicie, la altura mínima es de 10 metros y la máxima de 100 msnm., su superficie es plana con pendientes menores. Tiene un caudaloso río, el Candelaria. La región está integrada al sistema hidrológico GrijalvaUsumacinta, la cual comprende la corriente del río Candelaria que nace en el departamento del Petén (Guatemala), con el nombre de San Pedro, y desemboca en la Boca de Pargos, en la Laguna del Carmen. Una vez obtenidos los frutos, estos se llevaron al Laboratorio de Gastronomía del Instituto Tecnológico Superior de Escárcega (ITSE), el cual se encuentra ubicado en la ciudad de Escárcega, perteneciente al municipio del mismo nombre.

Elaboración de licor de piñuela

En laboratorio se procedió a la elaboración de licor de piñuela que consiste en las siguientes etapas:

Procedimiento para la elaboración de licor mediante el método de maceración. Como ya ha quedado descrito en el marco teórico el método de maceración; se explica a continuación, paso a paso como obtener licor de piñuela por medio de maceración alcohólica; ya que era preponderante que la fruta no perdiera sus propiedades organolépticas al momento de darle su transformación en licor:

Recolección de la fruta preferentemente en contenedores pequeños de plástico para que el producto no se maltrate.

Selección de la fruta que ha alcanzado su madurez fisiológica

Lavado con agua tibia, para desprender impurezas.

Extracción de la pulpa de la baya.

Colocación de la pulpa en contenedores inoxidables y se lava.

La pulpa limpia se colocó en un contenedor de vidrio

Se le agregó alcohol 100\% puro de caña

Se colocó en un lugar obscuro y se dejó macerando por un periodo de 45 días. En un recipiente se hirvió agua con azúcar para convertirlo en un jarabe no muy dulce.

Extracción del líquido del contenedor no sin antes tamizarlo perfectamente.

En ebullición, se retiró el jarabe del calor y se vertió en un nuevo recipiente previamente esterilizado junto con la solución obtenida de la maceración.

La mezcla de jarabe y la solución obtenida de la maceración se dejaron enfriar. Se seleccionó el envase definitivo y se esterilizó.

Se filtró el licor obtenido de todo el proceso anterior, utilizando papel filtro. Finalmente, el líquido se colocó en el envase definitivo, cerrado herméticamente y etiquetado

Se dejó reposar por espacio de tres meses entes de su consumo. 
Evaluación del grado de aceptación del licor de piñuela

La evaluación del grado de aceptación del licor de piñuela consistió en la selección de un panel de 50 personas, quienes previamente fueron semientrenados como se ha reportado en otros estudios (Pedrero y Pangborn, 1989). El licor fue presentado en vasos desechables de $200 \mathrm{ml}$., al que se le agregaron $50 \mathrm{ml}$ de licor. Se permitió a cada panelista probar las muestras tantas veces como lo deseara. Cada panelista califico al licor por su sabor, color y olor. Por lo que cada característica organoléptica se valoró de acuerdo a la escala de Likert.

\section{Resultados y Discusión}

Como resultado de la caracterización físico-química del licor de piñuela, se observó que el $\mathrm{pH}$ de este licor es de 3.5 considerando que un $\mathrm{pH}$ inicial debe estar por debajo de 3 y a medida que este sube, la acidez disminuye. Por lo que el resultado indica que su grado de acidez va de moderadamente ácido a ligeramente ácido. De igual forma la medición de los grados de alcohol fue de 31 y 15.7 de grados brix. Los resultados coinciden con otros estudios, Gómez et al. (2005) en su estudio de licor de mora a distintas concentraciones de la pulpa, reportó que su pH era de 3.15 a 3.2, mientras que sus grados brix daban valores de 13 a 26, y la concentración de alcohol va desde los 5 a los 7 . Por lo que se considera que la concentración de alcohol en el licor de piñuela es alto.

Por otro lado, la aceptabilidad indica que más del $80 \%$ de los panelistas están de acuerdo a totalmente de acuerdo satisfactoriamente con el sabor, color y olor del licor (Fig. 1). En la figura se puede observar que el sabor fue la característica principal que determinó su aceptabilidad. En los comentarios de los panelistas expresaron que la concentración de alcohol es de ligera a moderada. Gutiérrez-Jiménez et al. (2009) en su estudio manifestó que el sabor en un licor es significativo para su aceptación, mientras que Alvarez et al. (2002) expresó que en el caso del licor de mandarina la característica determinante fue el olor. 


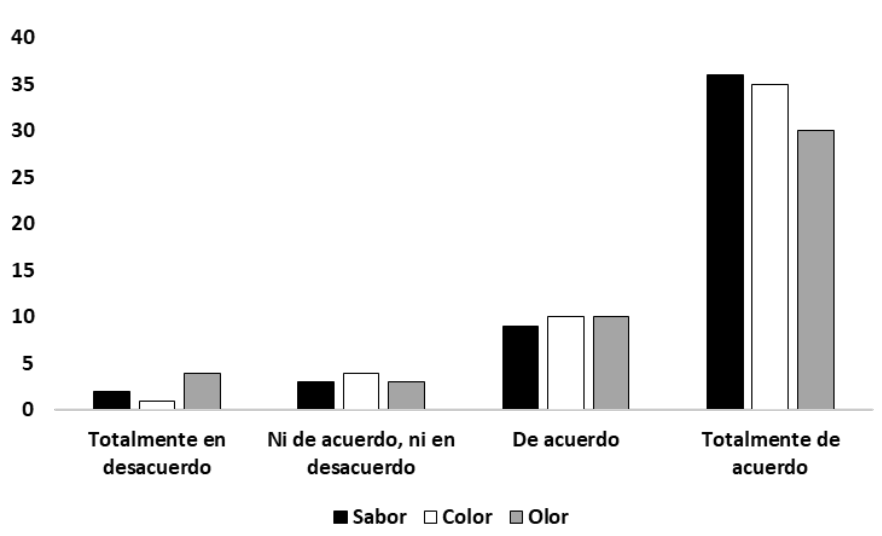

\section{Conclusiones}

Después del proceso investigativo y de haber confrontado datos de investigaciones similares, se encontró respuesta a la pregunta de investigación y se confirma nuestra hipótesis, si se puede obtener licor a partir del procesamiento artesanal del fruto de la piñuela en la región de Escárcega, en un periodo de 6 meses. Los objetivos se cumplieron; ya que se obtuvo el licor del fruto de la piñuela de forma artesanal, para consumo regional y a la vez se investigó sobre su uso y aprovechamiento para promover su consumo regional. Entre una de las actividades no consideradas en el estudio, sin embargo se realizó, fue la elaboración de trípticos para difundir entre la comunidad del Instituto Tecnológico Superior de Escárcega, las alternativas para el aprovechamiento del fruto de la piñuela. Finalmente, es importante considerar a este fruto como parte de un crecimiento en la industria alimentaria de la región, ya que por sus características naturales se le considera como un fuerte producto potencial para ser aprovechado por la comunidad local.

\section{Referencias}

1. Alvarez, M. J. M., Gutiérrez, G., Graterol, A., \& Belén, D. R. (2002). Evaluación de un licor dulce acondicionado con cáscaras de mandarina. Revista Científica de la Facultad de Ciencias Veterinarias, 12(4), 271-278.

2. Coe, F. G. \& Anderson, G. J. (1999). Ethnobotany of the Sumu (Ulwa) of Southeastern Nicaragua and comparitions with Miskitu plant lore. Econ Bot 53: 363 - 386.

3. Edouard F. (2005). Pita: renacimiento de la fibra natural más resistente pp 36-41. En La Riqueza de los bosques mexicanos más allá de la madera: experiencias de comunidades rurales. López, C., Chanfón S., Segura G. [Eds]. Secretaría de Medio Ambiente y Recursos Naturales (Semarnat), Centro de Educación y Capacitación para el Desarrollo Sustentable (Cecadesu), Comisión Nacional Forestal, Programa de 
Desarrollo Forestal Comunitario (Procymaf II), Center for International Forestry Research (CIFOR). México 201 pp.

4. Gómez, Á. M., Gómez, J. K. L., \& Cardozo, C. J. M. (2005). Licor de mora de castilla (Rubus glaucus Benth) con diferentes porcentajes de pulpa. Revista Facultad Nacional de Agronomía-Medellín, 58(2), 2963-2973.

5. Gutiérrez-Jiménez, L. C., González-Herrera, S. M., \& Soto-Cruz, O. (2009). Desarrollo y evaluación sensorial de un licor a base de mezcal. XIII Congreso Nacional de Biotecnología y Bioingeniería y VII SImposio Internacional de Producción de Alcoholes y Levaduras.

6. Hornung-Leoni, C. T. (2011). Avances sobre usos etnobotánicos de las Bromeliaceae en Latinoamérica. Boletin Latinoamericano y del Caribe de plantas Medicinales y Aromaticas, 10(4), 297-314.

7. Pedrero, D. L. \& Pangborn, R. M.(1989). Evaluación sensorial de los alimentos: métodos analíticos. México: Alhambra Mexicana. 248 p.

8. Pérez, P. M. (2009). Biblioteca Digital de la Medicina Tradicional Mexicana.

9. Sandoval-Bucio, E. N., Flores-Cruz, M. \& Martínez-Bernal A. (2004). Bromelias útiles de México. Cactáceas Suculentas Mexicanas 49: 100 - 115. 